\title{
Real-time Sensorless Estimation of Position and Force for Solenoid Actuators
}

\author{
Sakahisa Nagai* ${ }^{*}$ Student Member, Takahiro Nozaki ${ }^{* *}$ Member \\ Atsuo Kawamura* Fellow
}

(Manuscript received Feb. 12, 2015, revised May 6, 2015)

\begin{abstract}
In various fields, small actuation systems are required to aid human activities in a narrow space and to realize fine motions. Although some miniature actuators have been developed, the size of the sensors attached to them prevents the miniaturization of the systems. In this paper, a sensorless actuation system with a compact solenoid actuator is proposed. An input signal including the AC and DC components is used. The inductance and position are estimated from the AC component. The DC component is applied in order to drive the solenoid actuator. Simulations and experiments concerning frequency characteristics and simultaneous estimation are conducted to verify the validity of the proposal. From the results of the frequency characteristics, the position and force estimation are accurately achieved up to $10 \mathrm{~Hz}$ frequency. As a result of the simultaneous estimation, the position and force are simultaneously estimated in real time. This proposal is useful, because a small solenoid actuation system whose position and force are simultaneously estimated in real time without the need for any position and force sensors is realized.
\end{abstract}

Keywords: sensorless, solenoid actuator, real-time estimation, position and force simultaneous estimation

\section{Introduction}

Recently, emphasis has been put on communication techniques based on haptic information. Two examples are illustrative: in robotics and in the medical field. In robotics, to realize interactions between humans and their environment, robots that are used in the human environment have been developed. Environmental information is necessary for the robots to be able to adapt to the environment. By employing haptic information, human motion and environmental information can be analyzed ${ }^{(1)}$. In the medical field, haptic information is required for surgical teleoperation systems ${ }^{(2)}$. In conventional surgical teleoperation systems, operators cannot feel the softness of an organ, as only visual information is used. Therefore, operators often injure the organ. Haptic information enables the operators to receive tactile feedback regarding the organ, which reduces the number of the accidents. As stated above, haptic communication techniques are useful in various fields.

A tactile display is a haptic application that enables us to receive tactile feedback regarding the surface of a remote object $^{(3)(4)}$. This display typically consists of many actuators such as dielectric elastomer actuators ${ }^{(3)}$ and pneumatic actuators ${ }^{(4)}$. To represent the object's surface accurately, highdensity integration of actuators, provided by miniaturization, is required.

Small actuators are required not only for the development of a tactile display but also for supporting human activities

\footnotetext{
${ }^{*}$ Division of Electrical and Computer Engineering, Yokohama National University

79-5, Tokiwadai, Hodogaya-ku, Yokohama 240-8501, Japan

** Department of System Design Engineering, Keio University

3-14-1, Hiyoshi, Kohoku-ku, Yokohama 223-8522, Japan
}

in narrow spaces and for the realization of fine motion. For example, a globular magnetic actuator with four shapememory-alloy coils that can scan a complex pipe has been developed ${ }^{(5)}$. An XYZ stage consisting of piezo actuators has been researched to realize microscopic manipulation ${ }^{(6)}$.

Both position information and force information for an actuator are important to control its motion. For example, a bilateral control, which can communicate haptic information, requires simultaneous position information and applied force information ${ }^{(7)}$; by obtaining this information, various fine motion control methods can be applied to the actuator.

Because certain sensors are typically attached to the actuator to obtain this information, the system is large. Therefore, a sensorless actuation is an effective method for miniaturizing the system. As examples of sensorless actuation, positionsensorless controls on a linear tubular motor ${ }^{(8)}$ and an interior permanent magnet synchronous motor ${ }^{(9)}$ were studied. Removing the sensors provides other advantages such as reducing costs and frequency of maintenance.

A solenoid actuator is used to realize a small actuation system in this paper. Solenoid actuators are used in valves and switches. A braking system consisting of a solenoid actuator has been introduced ${ }^{(10)}$. The main features of the solenoid actuators are reduced cost, simple structure, and high output with a small volume.

The methods used to estimate the position (without using position sensors) for a solenoid actuator are divided into two main groups. The first estimation method uses a pulse width modulation (PWM) voltage input ${ }^{(11)(12)}$. The inductance of the solenoid is calculated based on the voltage and current responses. The inductance has a relation with the mover position; the position can be estimated using the relation. However, the PWM waveform includes harmonics, which cause 
undesirable effects such as unpleasant sounds and damage to the electronic circuits. I. Dülk et al. studied simultaneous estimation of the position and applied force, although the values of the position and force were discrete ${ }^{(12)}$. It is desirable that the estimated values be continuous when controlling the position and force.

The second estimation method uses an AC input ${ }^{(13)}$. The $\mathrm{AC}$ phase is measured to estimate the inductance. The position is determined by the relation with the inductance. By inserting a capacitor, resonance is utilized to realize accurate position estimation. However, two wires are required to estimate and drive the mover. To miniaturize the system, the number of the signal wires should be reduced. In addition, the resonant frequency was $125 \mathrm{~Hz}$ in the experiment conducted by S.-T. Wu et al.; therefore, the time required for the estimation was too long. A short estimation period is required to control the motion.

In this paper, simultaneous estimation of the mover position and applied force for a compact solenoid actuator is proposed. The input signal, which includes an AC component and a DC component, is applied to the solenoid actuator using one wire. The AC component is used for position estimation and the DC component is used for force generation. The characteristics of this proposal are as follows:

- Small solenoid actuation system.

- Lower harmonics.

- Real-time estimation.

- Simultaneous estimation of both position and force.

Simulations and experiments are conducted to verify the validity of this proposal.

This paper is organized as follows. Section 2 describes the model of the solenoid actuator. Section 3 describes the principles of sensorless position estimation. Section 4 describes the principles of sensorless force estimation. The characteristics of the solenoid actuator under test are presented in Sect. 5 The simulation results are presented in Sect. 6. The experimental results are presented in Sect. 7. Finally, the paper is concluded in Sect. 8 .

\section{Modeling of Solenoid Actuators}

This section describes the model of the solenoid actuator. A solenoid actuator consists of a mover, stator, coil, and spring, as shown in Fig. 1. The stator and mover are made of metal that has a high magnetic permeability, such as steel.

When current flows through the solenoid actuator, a magnetic field is generated. The magnetic coenergy $W_{\text {mag }}$ is

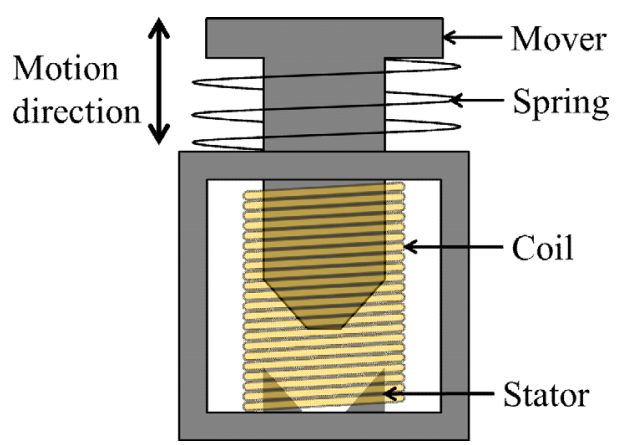

Fig. 1. Structure of solenoid actuator expressed as

$$
W_{\mathrm{mag}}=\frac{1}{2} L(x, i) i^{2},
$$

where $L, x$, and $i$ denote the coil inductance, mover position, and current, respectively. By differentiating Eq. (1), the magnetic force $F_{\text {mag }}$ is calculated as

$$
F_{\text {mag }}=\frac{\alpha}{2} \frac{\partial L(x, i)}{\partial x} i^{2},
$$

where $\alpha$ denotes the adjustment coefficient. The details of this effect are described in Appendix 1. The current $i$ consists of AC and DC components. The AC component $i_{\mathrm{ac}}$ is much smaller than the DC component $I_{\mathrm{dc}}$. Therefore, $F_{\text {mag }}$ is controlled by modulating $I_{\mathrm{dc}}$. Figure 2 shows the force applied to the mover: $F_{\text {mag }}, F_{\text {spr }}$, and $F_{\text {ext }} . F_{\text {spr }}$ is the elastic force generated by the spring. It is calculated as

$$
F_{\mathrm{spr}}=k\left(x_{0}-x\right), \cdots \ldots \ldots \ldots \ldots \ldots \ldots \ldots \ldots \ldots \ldots \ldots \ldots \ldots \ldots \ldots \ldots
$$

where $k$ denotes the elastic coefficient of the spring. The origin of the $x$ axis is set at the lowest point. The point $x_{0}$ is the equilibrium point at which gravity balances the elastic force when the DC current $I_{\mathrm{dc}}$ does not flow. The length of the gap between the mover and the stator is equal to the mover position. $F_{\text {ext }}$ is an external force such as a friction force and a force applied by a human. The motion equation is derived as

$$
m \ddot{x}=F_{\mathrm{spr}}-F_{\mathrm{mag}}-F_{\mathrm{ext}}
$$

where $m$ denotes the mass of the mover.

\section{Principle of Sensorless Position Estimation}

The circuit equation is derived as

$$
v=R i+\frac{d \Psi}{d t}, \cdots
$$

where $v, R, \Psi$, and $t$ denote the voltage, internal resistance, linkage flux, and time, respectively. On the right-hand side of Eq. (5), the first term describes the voltage drop due to the internal resistance. The second term represents the induced electromotive voltage. The linkage flux $\Psi$ is expressed as

$$
\Psi=L i
$$

Thus, Eq. (5) can be rewritten as

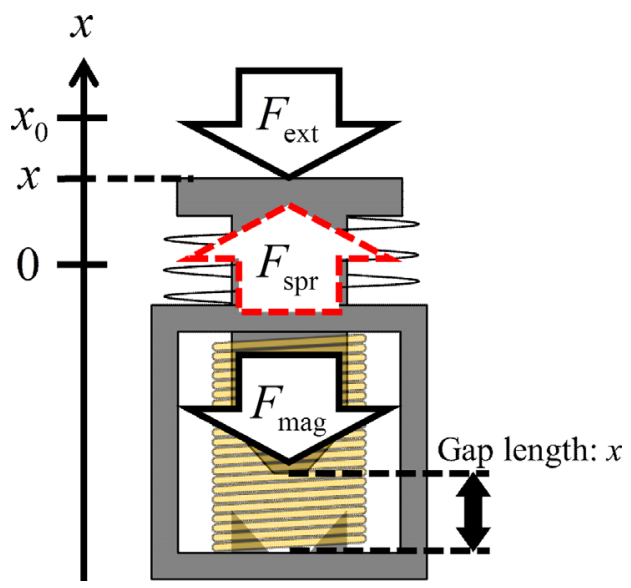

Fig. 2. Force applied to mover 


$$
v=R i+\frac{d L}{d t} i+L \frac{d i}{d t}
$$

When a signal that includes AC and DC components is applied to the solenoid actuator, the voltage and current are represented by

$$
\begin{aligned}
& v=v_{\mathrm{ac}}+V_{\mathrm{dc}}, \\
& i=i_{\mathrm{ac}}+I_{\mathrm{dc}},
\end{aligned}
$$

where the subscripts "ac" and "dc" denote the AC and DC components. $v_{\mathrm{ac}}$ and $V_{\mathrm{dc}}$ are the voltage. Putting Eqs. (8) and (9) into Eq. (7) yields

$$
\begin{aligned}
v_{\mathrm{ac}}+V_{\mathrm{dc}}= & R\left(i_{\mathrm{ac}}+I_{\mathrm{dc}}\right) \\
& +\frac{d L}{d t}\left(i_{\mathrm{ac}}+I_{\mathrm{dc}}\right)+L \frac{d i_{\mathrm{ac}}}{d t} .
\end{aligned}
$$

If the $\mathrm{AC}$ and $\mathrm{DC}$ components are assumed to be separated, Eq. (10) can be divided into two equations as

$$
\begin{aligned}
v_{\mathrm{ac}} & =R i_{\mathrm{ac}}+\frac{d L}{d t} i_{\mathrm{ac}}+L \frac{d i_{\mathrm{ac}}}{d t}, \\
V_{\mathrm{dc}} & =R I_{\mathrm{dc}}+\frac{d L}{d t} I_{\mathrm{dc}} \cdot \cdots \cdots
\end{aligned}
$$

From Eq. (12), dividing both sides by $I_{\mathrm{dc}}$ yields

$$
R+\frac{d L}{d t}=\frac{V_{\mathrm{dc}}}{I_{\mathrm{dc}}}
$$

Putting Eq. (13) into Eq. (11) yields

$$
v_{\mathrm{ac}}=\frac{V_{\mathrm{dc}}}{I_{\mathrm{dc}}} i_{\mathrm{ac}}+L \frac{d i_{\mathrm{ac}}}{d t} \text {. }
$$

When $i_{\mathrm{ac}}=\bar{i}_{\mathrm{ac}} \cos (\omega t+\phi)$, the circuit equation can be solved as

$$
v_{\mathrm{ac}}=\bar{v}_{\mathrm{ac}} \cos (\omega t+\phi+\theta),
$$

where $\bar{i}_{\mathrm{ac}}, \omega, \phi, \bar{v}_{\mathrm{ac}}$, and $\theta$ denote the AC current amplitude, angular velocity, initial phase of the AC current, AC voltage amplitude, and the phase difference between $\mathrm{AC}$ voltage and current, respectively. $\bar{v}_{\text {ac }}$ and $\theta$ are calculated as

$$
\begin{aligned}
& \bar{v}_{\mathrm{ac}}=\bar{i}_{\mathrm{ac}} \sqrt{\left(\frac{V_{\mathrm{dc}}}{I_{\mathrm{dc}}}\right)^{2}+(\omega L)^{2},} \\
& \theta=\tan ^{-1} \frac{\omega L}{\left(\frac{V_{\mathrm{dc}}}{I_{\mathrm{dc}}}\right)}, \ldots \ldots \ldots \ldots
\end{aligned}
$$

Solving $L$ in Eq. (16) yields

$$
L=\frac{1}{\omega} \sqrt{\left(\frac{\bar{v}_{\mathrm{ac}}}{\bar{i}_{\mathrm{ac}}}\right)^{2}-\left(\frac{V_{\mathrm{dc}}}{I_{\mathrm{dc}}}\right)^{2}}
$$

This equation indicates that the inductance of the solenoid can be obtained from the AC and DC voltage and current. The inductance has a relation with the mover position. By identifying the relation in advance, the position can be estimated from the inductance.

\section{Principle of Sensorless Force Estimation}

The motion equation is given in Eq. (4). The elastic force $F_{\text {spr }}$ in Eq. (3) and the inertial force $m \ddot{x}$ can be estimated from the estimated position. The magnetic force $F_{\text {mag }}$ in Eq. (2) can be derived from the DC current. Therefore, the external force $F_{\text {ext }}$ can be estimated as

$$
F_{\text {ext }}=F_{\text {spr }}-F_{\text {mag }}-m \ddot{x} \text {. }
$$

\section{Characteristics of Solenoid Actuator under Test}

This section presents the characteristics of the solenoid actuator that is used in the simulations and experiments. Figure 3 shows the compact solenoid actuator, DS-05A, J100001, made by CKD Corporation. The size is $\mathrm{H}: 43.5 \mathrm{~mm} \times$ $\mathrm{W}: 15 \mathrm{~mm} \times \mathrm{D}: 10 \mathrm{~mm}$. The mass of the mover is $4.80 \mathrm{~g}$. The internal resistance is $20 \Omega$. The elastic coefficient of the spring is $50 \mathrm{~N} / \mathrm{m}$. A laser sensor, CD22-15A, made by OPTEX FA Corporation, was used to measure the actual mover position.

The relation between the mover position and the inductance was measured by an LCR meter. The input voltage and frequency were set at $3 \mathrm{~V}$ and $1 \mathrm{kHz}$, respectively We assume that the relation can be described by a quadratic polynomial of the following form:

$$
x=a L^{2}+b L+c,
$$

where $a, b$, and $c$ are constants. Figure 4 shows the measured relation. The symbol " shows the measured points. The solid line shows a quadratic polynomial approximation. The parameters obtained are shown in Table 1.

Figure 5 shows the relation between DC current and elastic force in Eq. (3) on the non-contact condition. The symbol " represents the measured elastic force. The magnetic attractive force is represented in Eq. (2). Because the DC current is small, the inductance $L$ can be assumed to be a function of only the mover position $x . \partial L(x) / \partial x$ is calculated from Eq. (20) as

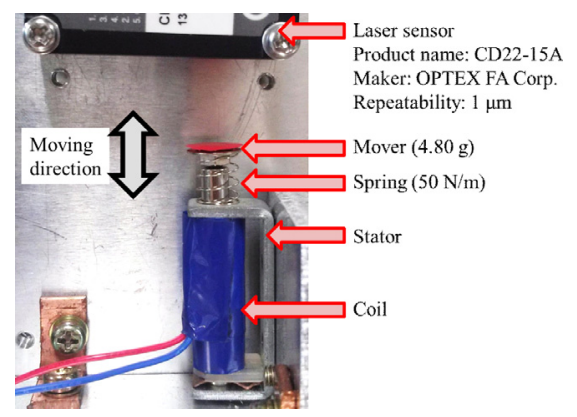

Fig. 3. Solenoid actuator

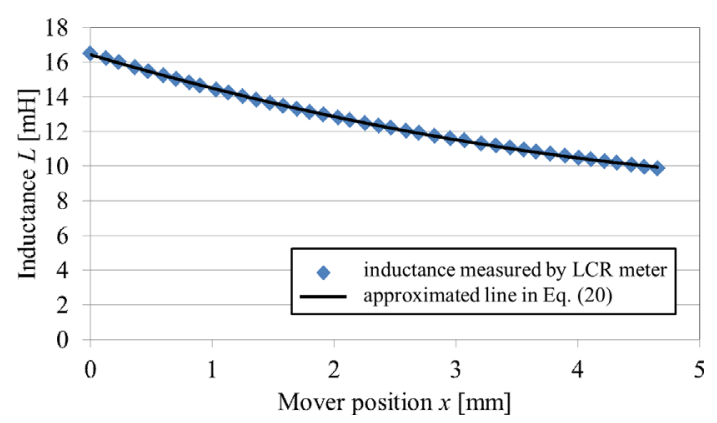

Fig. 4. Relation between mover position and inductance

Table 1. Parameters of solenoid actuator

\begin{tabular}{ccc}
\hline coefficients of relation & $a$ & 0.0335 \\
between mover position & $b$ & -1.56 \\
and inductance & $c$ & 17.8 \\
\hline adjustment coefficient & $\alpha$ & 4.55 \\
\hline
\end{tabular}




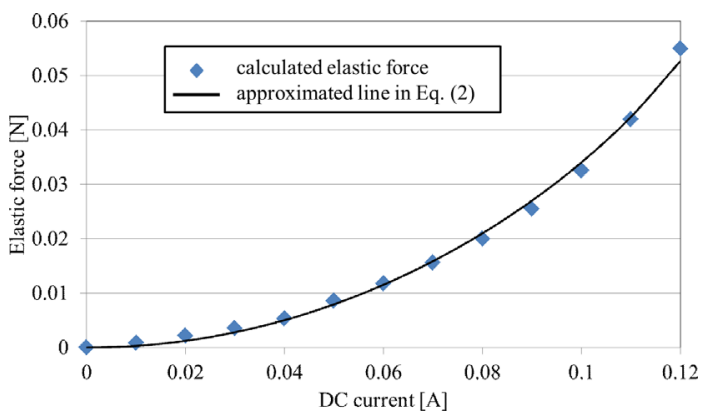

Fig. 5. Magnetic force characteristics (no load)

$$
\frac{\partial L(x)}{\partial x}=-\frac{1}{\sqrt{b^{2}-4 \cdot a \cdot(c-x)}} \cdot \cdots \ldots \ldots \ldots \ldots \ldots
$$

The solid line in Fig. 5 shows the approximation using the same equation form as in Eq. (2). The parameter $\alpha$ was determined as shown in Table 1.

\section{Simulations}

This section describes our simulation results. To confirm the validity of the proposal, several simulations were conducted.

6.1 Position Estimation The frequency characteristics of the position estimation method were confirmed by simulations. The DC current was used to displace the mover and create a sinusoidal motion. The AC component (whose voltage and frequency were $3 \mathrm{~V}$ and $1 \mathrm{kHz}$, respectively) was constantly input. Figure 6 shows the simulation result of the position estimation at various frequencies. The vertical axis represents the error ratio of the position estimation, which is calculated as

$$
E_{\mathrm{p}}=10 \log _{10} \frac{\bar{x}}{\overline{\hat{x}}},
$$

where $\bar{x}$ and $\overline{\hat{x}}$ denote the amplitude of the actual position and the estimated position, respectively. The symbol " $"$ " represents the calculated points. Up to a frequency of roughly $10 \mathrm{~Hz}$, the position can be estimated accurately. The position estimation accuracy worsens at higher frequencies than the natural frequency of $\sqrt{k / m} / 2 \pi=16.2 \mathrm{~Hz}$.

6.2 Real-time Characteristics The estimation period is confirmed using a simulation. The position oscillates by modulating the DC component at $1 \mathrm{~Hz}$. The estimated position is compared to the actual position. The root-meansquare (RMS) error is calculated from the actual and estimated position over one period by shifting the estimated position as

$$
\mathrm{RMS}_{\text {error }}=\sqrt{\frac{1}{N} \sum_{k=0}^{N}\{x(k)-\hat{x}(k+\Delta t)\}^{2}}, \cdots \cdots
$$

where $N$ and $\Delta t$ denote the number of the data points in a period and the shifting time, respectively. $\Delta t$ being at the minimum point of the RMS error indicates that the actual position $x(k)$ is similar to the estimated position $\hat{x}(k)$, with a shift of $\Delta t$. In short, $\Delta t$ represents the time required for position estimation. Figure 7 shows the calculated RMS error when changing $\Delta t$. The symbol " shows the points calculated using Eq. (23). The smallest RMS error is $0.12 \mu \mathrm{m}$ at

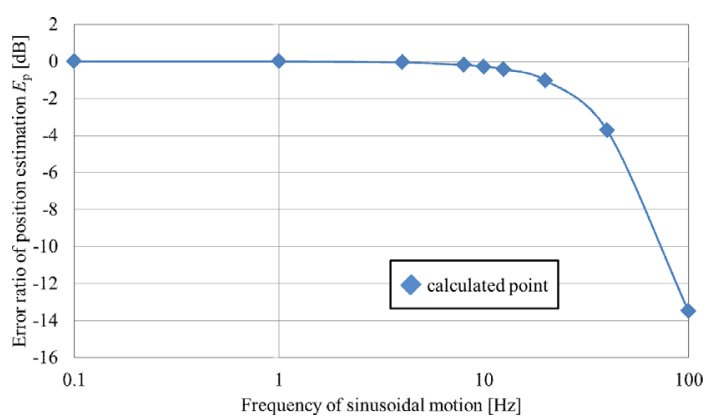

Fig. 6. Frequency characteristics of position estimation (simulation)

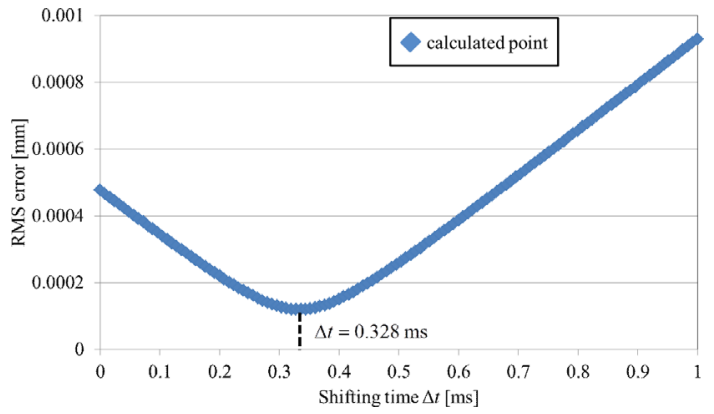

Fig. 7. RMS error calculation for real-time characteristics (simulation)

$\Delta t=0.328 \mathrm{~ms}$. This result confirms that the position can be estimated within $0.328 \mathrm{~ms}$.

6.3 Force Estimation The frequency characteristics of the force estimation were confirmed by simulations. It is assumed that the mover makes contact with an object, which is represented as a spring-damper model. The external force is calculated as

$$
F_{\text {ext }}=K_{\mathrm{e}}\left(x_{\mathrm{e}}-x\right)-D_{\mathrm{e}} \dot{x},
$$

where $K_{\mathrm{e}}, D_{\mathrm{e}}$, and $x_{\mathrm{e}}$ represent the elastic and viscous damping coefficient of the contact object, and the contact point with the object, respectively. In this simulation, the contact environment consists of a hard object whose coefficients $K_{\mathrm{e}}$ and $D_{\mathrm{e}}$ are set at $1000 \mathrm{~N} / \mathrm{m}$ and $0.1 \mathrm{Ns} / \mathrm{m}$. The DC current oscillates under the condition which the mover consistently comes into contact with the object. Figure 8 shows the simulation result of the force estimation at various frequencies. The vertical axis represents the error ratio of the force estimation, which is calculated as

$$
E_{\mathrm{f}}=10 \log _{10} \frac{\bar{F}_{\text {ext }}}{\overline{\hat{F}}_{\text {ext }}}, \cdot \cdots
$$

where $\bar{F}_{\text {ext }}$ and $\overline{\hat{F}}_{\text {ext }}$ denote the amplitude of the actual external force and the estimated force, respectively. The symbol " " represents the calculated points. Force estimation is possible up to a frequency of $10 \mathrm{~Hz}$. At higher frequencies, the position estimation worsens, as shown in Fig. 6. Therefore, the accuracy of the elastic force calculation in Eq. (3) deteriorates. In addition, the magnetic force calculation depends on the position, in terms of $\partial L / \partial x$; therefore, the accuracy of the force estimation worsens.

6.4 Simultaneous Estimation Figures 9 and 10 show the simulation results when the position and force are simultaneously estimated. The solid line and broken line in Fig. 9 


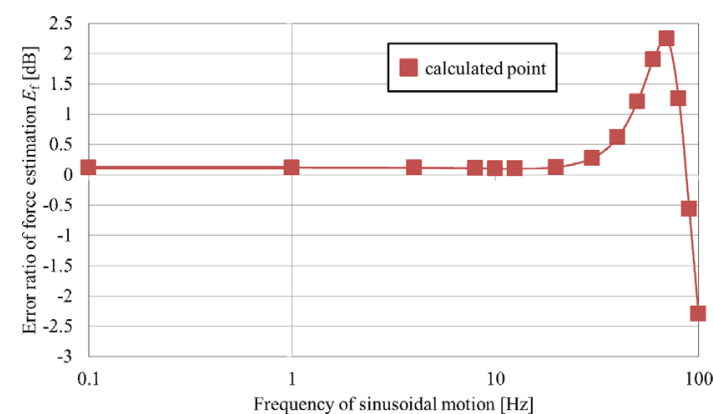

Fig. 8. Frequency characteristics of force estimation (simulation)

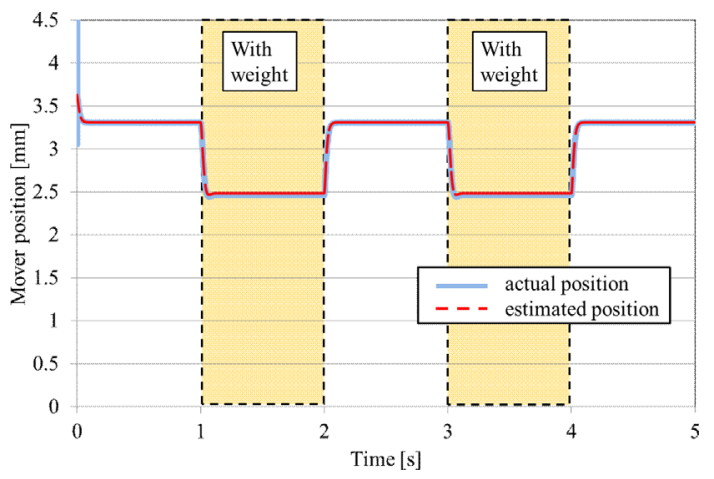

Fig. 9. Position response of simultaneous position and force estimation with periodic external force (simulation)

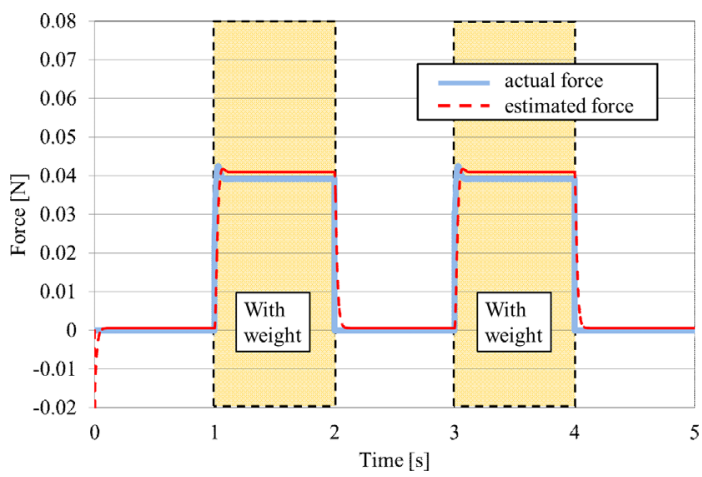

Fig. 10. Force response of simultaneous position and force estimation with periodic external force (simulation)

represent the actual position and estimated position, respectively. The solid line and broken line in Fig. 10 represent the actual force and estimated force, respectively. Here, we assume that the external force is $4.00 \mathrm{~g}$ in weight $(39.2 \mathrm{mN})$ and that the DC component is constantly input. The position and force are accurately estimated in real-time. The small error in the force estimation is due to the position estimation error.

\section{Experiments}

This section describes our experimental results. Real-time estimation experiments for the mover position and force were conducted to verify the validity of the proposal.

7.1 Experimental Setup The experimental system is shown in Fig. 11. The controller generated the input signal. The D/A converter output a staircase wave. The signal was input to the solenoid actuator via a low-pass filter and an operational amplifier. The voltage and current were detected by the A/D converter. A high speed digital control system called

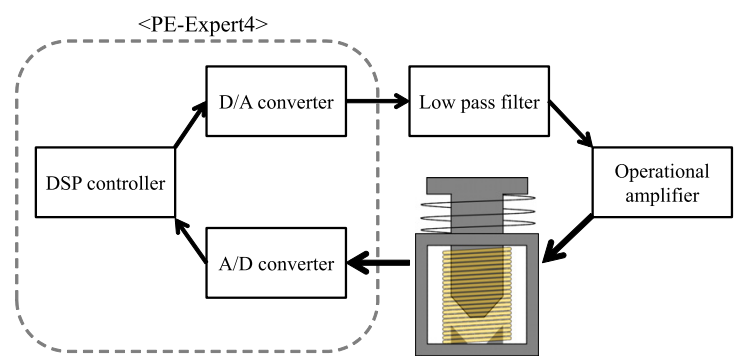

Fig. 11. Experimental system

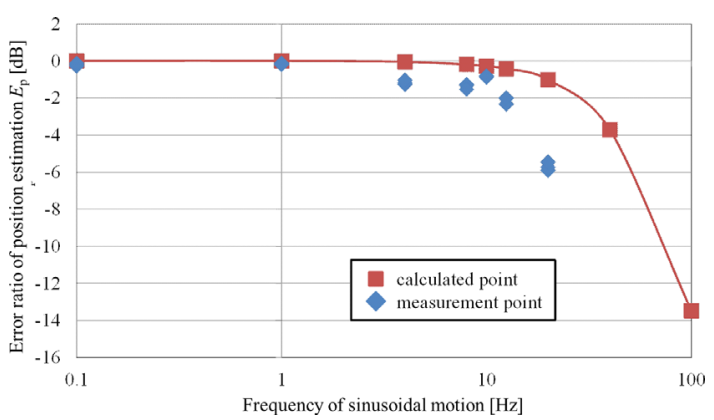

Fig. 12. Frequency characteristics of position estimation (experiment)

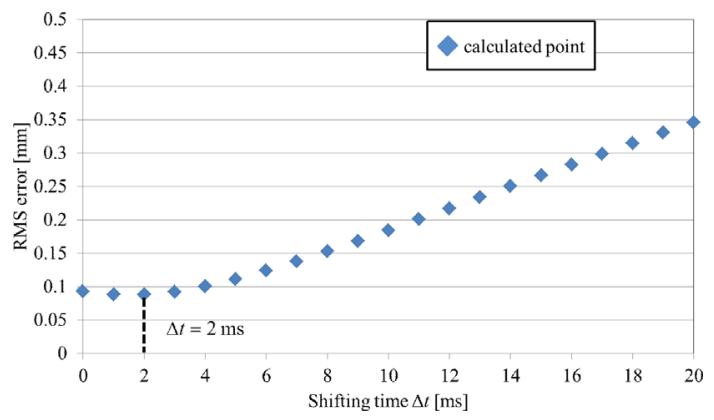

Fig. 13. RMS error calculation for real-time characteristics (experiment)

PE-Expert4, made by Myway Plus Corporation, was used. The main CPU is a digital signal processor. The A/D converter sampled the data every $5 \mu$ s. The $\mathrm{D} / \mathrm{A}$ converter output a signal every $20 \mu \mathrm{s}$.

7.2 Position Estimation The position estimation experiment was conducted under the non-contact condition. Figure 12 shows the experimental results of the position estimation. The vertical axis represents the error ratio of the position estimation calculated using Eq. (22). The symbol "\$" shows the measured points. The symbol " $\square$ " represents the simulation result shown in Fig. 6. Position estimation could be achieved at more than $-2 \mathrm{~dB}$ accuracy up to $10 \mathrm{~Hz}$.

7.3 Real-time Characteristic The real-time characteristics were measured by changing the DC component. The RMS error was calculated using Eq. (23); the result is shown in Fig. 13. The symbol " represents the calculated points. The minimum value of the RMS error is $0.08815 \mathrm{~mm}$ at $\Delta t=2 \mathrm{~ms}$. In short, real-time estimation of the position can be achieved within $2 \mathrm{~ms}$. The difference between the simulation and experimental results is due to the difference of the sampling time. The experimental data was derived by using software called PE-View X, made by Myway Plus Corporation. The sampling time was $1 \mathrm{~ms}$, which was longer than the 


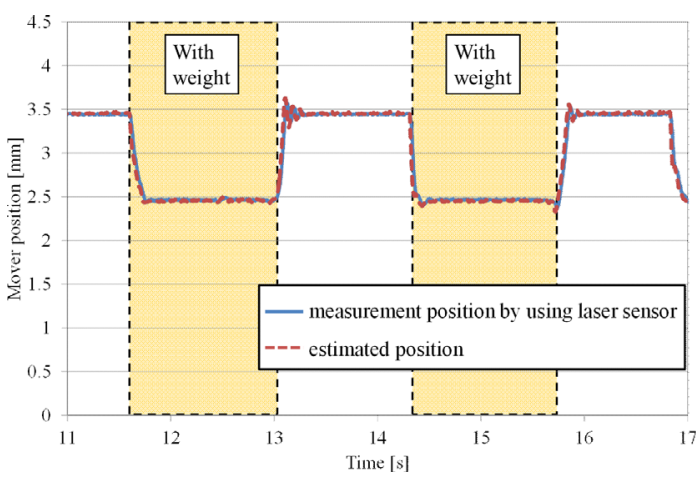

Fig. 14. Position response of simultaneous position and force estimation with periodic external force (experiment)

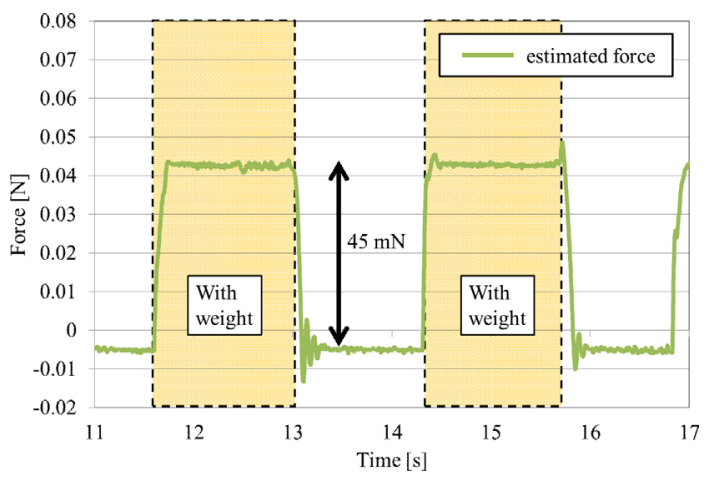

Fig. 15. Force response of simultaneous position and force estimation with periodic external force (experiment)

sampling time used in the simulations.

7.4 Simultaneous Estimation The position and force were simultaneously estimated. The external force was periodically applied by putting a $4.00 \mathrm{~g}$ weight $(39.2 \mathrm{mN})$ on the mover. As the mass of the mover $m$ was very small, the inertial force $m \ddot{x}$ was assumed to be zero. Figures 14 and 15 shows the experimental results of the simultaneous estimation of the mover position and the external force. The solid line and broken line in Fig. 14 represent the position measured by the laser sensor and the estimated position, respectively. The solid line in Fig. 15 represents the estimated force. The position was accurately estimated in real-time. The force was estimated at $45 \mathrm{mN}$, which is slightly larger than the force of gravity on the weight (i.e., $39.2 \mathrm{mN}$ ). This error comes from the error in the position estimation and the static friction.

\section{Conclusions}

This paper proposed real-time sensorless estimation for solenoid actuators. There are four advantages to using this technique: 1) compact size, 2) lower harmonics, 3) simultaneous estimation of position and force, and 4) real-time estimation. Simulations and experiments were conducted to confirm the frequency characteristics and real-time characteristics, and that simultaneous estimation was possible. In the experimental results for the frequency characteristics, the accuracy of the position estimation was confirmed to be more than $-2 \mathrm{~dB}$ at frequencies under $10 \mathrm{~Hz}$. As the experimental results of the real-time characteristics show, the proposed technique was able to successfully estimate the position within $2 \mathrm{~ms}$. In the experimental results for the simultaneous estimation, both the position and force were estimated at the same time; however, there were about $5 \mathrm{mN}$ force errors.

As mentioned above, the novelty of this proposal is the real-time simultaneous estimation of position and force for a compact solenoid actuator by inputting a signal with lower harmonics. This proposal is useful for fields that require compact actuation systems, such as tactile displays and pipe inspection devices.

\section{References}

( 1 ) S. Hyodo and K. Ohnishi: "A method for motion abstraction based on haptic information directionality and an application to haptic motion display system", IEEE Trans. Ind. Electron., Vol.56, No.5, pp.1356-1363 (2009)

( 2 ) W. McMahan, J. Gewirtz, D. Standish, P. Martin, J.A. Kunkel, M. Lilavois, A. Wedmid, D.I. Lee, and K.J. Kuchenbecker: "Tool contact acceleration feedback for telerobotic surgery", IEEE Trans. Haptics, Vol.4, No.3, pp.210220 (2011)

( 3 ) S. Yun, S. Park, B. Park, S.K. Park, H. Prahlad, P.V. Guggenberg, and K.-U. Kyung: "Polymer-based flexible visuo-haptic display", IEEE/ASME Trans. Mechatronics, Vol.19, No.4, pp.1463-1469 (2014)

( 4 ) C.-H. King, M.O. Culjat, M.L. Franco, J.W. Bisley, E. Dutson, and W.S. Grundfest: "Optimization of a pneumatic balloon tactile display for robot-assisted surgery based on human perception", IEEE Trans. Biomed. Eng., Vol.55, No.11, pp.2593-2600 (2008)

( 5 ) H. Yaguchi and N. Sato: "Globular magnetic actuator capable of free movement in a complex pipe", IEEE Trans. Magnetics, Vol.46, No.6, pp.13501355 (2010)

( 6 ) Y. Li and Q. Xu: "A totally decoupled piezo-driven xyz flexure parallel micropositioning stage for micro/nanomanipulation", IEEE Trans. Automation Science and Engineering, Vol.8, No.2, pp.265-279 (2011)

( 7 ) T. Nozaki, T. Mizoguchi, and K. Ohnishi: "Real-world haptics for motion realization", IEEJ Journal of Industry Applications, Vol.2, No.1, pp.1-6 (2013)

( 8 ) F. Cupertino, P. Giangrande, G. Pellegrino, and L. Salvatore: "End effects in linear tubular motors and compensated position sensorless control based on pulsating voltage injection", IEEE Trans. Ind. Electron., Vol.58, No.2, pp.494-502 (2011)

( 9 ) A. Matsumoto, M. Hasegawa, M. Tomita, and S. Doki: "Position sensorless control of IPMSMs using full-order flux observer based on an algebraic design method", IEEJ Journal of Industry Applications, Vol.2, No.3, pp.141149 (2013)

(10) M. Branciforte, A. Meli, G. Muscato, and D. Porto: "ANN and non-integer order modeling of ABS solenoid valves", IEEE Trans. Control Systems Technology, Vol.19, No.3, pp.628-635 (2011)

(11) J.-C. Renn and Y.-S. Chou: "Sensorless plunger position control for a switching solenoid", JSME International Journal Series C, Vol.47, No.2, pp.637645 (2005)

(12) I. Dülk and T. Kováscházy: "A sensorless method for detecting spool position in solenoid actuators", Carpathian Journal of Electronic and Computer Engineering, Vol.6, No.1, pp.36-43 (2013)

(13) S.-T. Wu and W.-N. Chen: "Self-sensing of a solenoid valve via phase detection", In Proc. of 2009 IEEE/ASME International Conference on Advanced Intelligent Mechatronics, pp.1165-1170 (2009)

\section{Appendix}

\section{Explanation of adjustment coefficient $\alpha$ based on magnetized mover and stator}

The measured force is several times larger than the theoretically predicted electromagnetic force, thus the authors introduce an adjustment coefficient $\alpha$ based on the properties of the magnetized mover and stator. The magnetic force $F_{\text {mag }}$ is divided into two parts as follows:

$$
F_{\text {mag }}=\frac{1}{2} \frac{\partial L}{\partial x} i^{2}-\frac{m_{1} m_{2}}{x^{2}}, \cdots \ldots \ldots \ldots \ldots \ldots \ldots
$$


where $m_{1}$ and $m_{2}$ denote the magnetization intensity of the mover and the stator, respectively. On the right-hand side of Eq. (A1), the first term is the force generated by the magnetic energy $W_{\mathrm{mag}}$; the second term is the elastic force generated by magnetizing the mover and the stator. Using the Taylor series expansion around the initial position $x_{0}$, Eq. (A1) can be rewritten as

$$
F_{\mathrm{mag}} \approx \frac{1}{2} \frac{\partial L}{\partial x} i^{2}-\frac{m_{1} m_{2}}{x_{0}^{2}}+2 \frac{m_{1} m_{2}}{x_{0}^{3}}\left(x_{0}-x\right) .
$$

On the right-hand side of Eq. (A2), the second term is constant. This term can be ignored by setting the initial position $x_{0}$ at the equilibrium position at which the mover weight, elastic force, and this term are balanced. Therefore, the magnetic force can be expressed as

$$
F_{\mathrm{mag}} \approx \frac{1}{2} \frac{\partial L}{\partial x} i^{2}+2 \frac{m_{1} m_{2}}{x_{0}{ }^{3}}\left(x_{0}-x\right) \ldots \ldots \ldots \cdots \cdots
$$

In Sect. 5, the magnetic force characteristics were measured using the balance between the elastic force and the magnetic force, as shown in Fig. 5, where the mover was not fixed. The relation between the current and the position can be calculated as

$$
\begin{aligned}
F_{\mathrm{spr}} & =k\left(x_{0}-x\right) \\
& =F_{\mathrm{mag}}=\frac{1}{2} \frac{\partial L}{\partial x} i^{2}+2 \frac{m_{1} m_{2}}{x_{0}{ }^{3}}\left(x_{0}-x\right) .
\end{aligned}
$$

Therefore,

$$
x_{0}-x=\frac{1}{2}\left(\frac{1}{k-2 \frac{m_{1} m_{2}}{x_{0}{ }^{3}}}\right) \frac{\partial L}{\partial x} i^{2} .
$$

Putting Eq. (A5) into Eq. (A3) yields

$$
F_{\text {mag }}=\frac{\alpha}{2} \frac{\partial L}{\partial x} i^{2}
$$

where

$$
\alpha=1+\frac{2 \frac{m_{1} m_{2}}{x_{0}{ }^{3}}}{k-2 \frac{m_{1} m_{2}}{x_{0}{ }^{3}}} .
$$

This value $\alpha$ was experimentally determined.

Sakahisa Nagai (Student Member) received the B.E. degrees in fac-

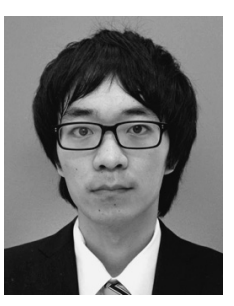
ulty of engineering from Yokohama National University, Kanagawa Japan, in 2014. He is now a master course student of Yokohama National University. He has belonged to the Kawamura laboratory since April 2013. His research interests include sensorless actuation and motion control.

Takahiro Nozaki (Member) received the B.E. degree in system de-

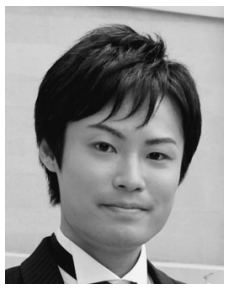
sign engineering and the M.E. and Ph.D. degrees in integrated design engineering from Keio University, Japan, in 2010, 2012, and 2014, respectively. After working at Yokohama National University, Yokohama, Japan, as an assistant professor for one year, he has been with Keio University, Yokohama, Japan, as an assistant professor since 2015.

Atsuo Kawamura (Fellow) received the Ph.D. degree in electrical en-

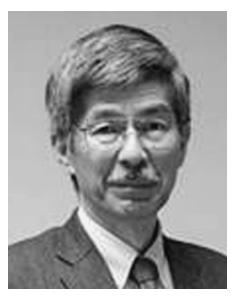
gineering from the University of Tokyo in 1981. After the five-year-stay at the University of MissouriColumbia as a faculty member, he joined the department of electrical and computer engineering at Yokohama National University in 1986, and now he is a professor. His interests are in the fields of power electronics, digital control, electric vehicles, train traction control and robotics. He received Transaction Paper Award from IEEE in 1988, 2001 and 2002, also from IEE of Japan in 1996. Dr. Kawamura is an IEEE Fellow, and Fellow of the IEE of Japan. 